MATHEMATICS OF COMPUTATION

Volume 73, Number 247, Pages 1565-1575

S 0025-5718(04)01649-7

Article electronically published on February 25, 2004

\title{
COUNTING PRIMES IN RESIDUE CLASSES
}

\author{
MARC DELÉGLISE, PIERRE DUSART, AND XAVIER-FRANÇOIS ROBLOT
}

\begin{abstract}
We explain how the Meissel-Lehmer-Lagarias-Miller-Odlyzko method for computing $\pi(x)$ can be used for computing efficiently $\pi(x, k, l)$, the number of primes congruent to $l$ modulo $k$ up to $x$. As an application, we computed the number of prime numbers of the form $4 n \pm 1$ less than $x$ for several values of $x$ up to $10^{20}$ and found a new region where $\pi(x, 4,3)$ is less than $\pi(x, 4,1)$ near $x=10^{18}$.
\end{abstract}

\section{INTRODUCTION}

In the 1870's, the German astronomer Meissel designed a method for computing the value of $\pi(x)$, the number of prime numbers up to $x$. The method has been improved by many authors since then. The most important improvement is due to Lagarias-Miller-Odlyzko LMO85 which obtained a method requiring $O\left(x^{2 / 3} / \log x\right)$ time and computed the value of $\pi\left(4 \cdot 10^{16}\right)$. Further improvements were obtained by the first author and Rivat DR96 with $O\left(x^{2 / 3} / \log ^{2} x\right)$ time and who computed $\pi\left(10^{18}\right)$. Finally, Gourdon, using ideas originating from LagariasMiller-Odlyzko, implemented a parallel version of the algorithm and computed, to date, values of $\pi(x)$ up to $4 \cdot 10^{22}$.

For $l$ and $k$ two relatively prime positive integers, one defines $\pi(x, k, l)$ as the number of prime numbers up to $x$ that are congruent to $l$ modulo $k$. Asymptotically the numbers $\pi(x, k, l)$ are all of same size, $\varphi(k)^{-1} x / \log x$. However it has been known for quite some time that there are more primes in the congruence classes that are nonquadratic residues modulo $k$ than in those that are. Heuristically, this bias can be explained from the fact that these classes contain more composite numbers than the latter since they contain all the squares (see also [RS94]).

For $k=4$, there are two classes, the numbers congruent to 1 modulo 4 , the quadratic residues, and the numbers congruent to 3 modulo 4 , the nonquadratic residues. In this setting Littlewood proved that (see [ng90 for the $\Omega_{ \pm}$notation)

$$
\pi(x, 4,3)-\pi(x, 4,1)=\Omega_{ \pm}\left(\frac{x^{1 / 2}}{\log x} \log \log \log x\right) .
$$

Therefore there are infinitely many sign changes for the function $\delta(x)=\pi(x, 4,3)-$ $\pi(x, 4,1)$. Define two disjoint subsets of the set of integers:

$$
\begin{aligned}
& \Delta^{+}=\{x \geq 2: \delta(x)>0\}, \\
& \Delta^{-}=\{x \geq 2: \delta(x)<0\} .
\end{aligned}
$$

Received by the editor November 7, 2001 and, in revised form, October 24, 2002.

2000 Mathematics Subject Classification. Primary 11Y40; Secondary 11A41.

Key words and phrases. Prime numbers, residue classes, Chebyshev's bias. 
For $A$ a subset of the positive integers, the logarithmic density $d(A)$ is defined as the following limit, if it exists:

$$
d(A)=\lim _{x \rightarrow \infty} \frac{1}{\log x} \sum_{\substack{a \in A \\ a \leq x}} \frac{1}{a} .
$$

Note that any set $A$ admitting a density in the usual sense admits also a logarithmic density, and the two densities are equal. However, there exist some sets (e.g., the set of numbers whose decimal expansion starts with 1) with a logarithmic density (in this example $\log 2 / \log 10$ ) but not having a density in the usual sense.

In [RS94], Rubinstein and Sarnak proved that under suitable generalization of Riemann Hypothesis (RH) both sets admit a logarithmic density. More exactly, they proved, conditionally under these assumptions, that

$$
d\left(\Delta^{+}\right)=0.99592 \ldots \text { and } d\left(\Delta^{-}\right)=0.00407 \ldots
$$

These results have been further generalized and improved in [FM00] and [BFHR01].

From the computational point of view, several people have been searching for a region containing elements of $\Delta^{-}$(see [Lee57, [BH78], [BFHR01]). So far, eight regions have been found and we have discovered a new region using the method described in this paper. See the last section for more details.

In this article, we will prove the following theorem:

Theorem 1. Let $x>0$, and let $k$ and $l$ be two relatively prime positive integers. There exists an algorithm which computes $\pi(x, k, l)$ in time $O\left(x^{2 / 3} / \ln ^{2} x\right)$.

Note that the computation time of this algorithm is exactly that of the algorithm for the computation of $\pi(x)$ given in DR96. Indeed, loops that ranged through the primes less than a given bound $B$ in the computation of $\pi(x)$ are now replaced by $\varphi(k)$ loops, one for each invertible class modulo $k$ ranging through the primes less than $B$ in that class. Therefore, the total number of operations stays the same. In particular, the running time does not depend on the values of $k$ or $l$. Of course, for fixed values of $x$ and $k$, the computation of all $\pi(x, k, l)$ where $l$ ranges through the $\varphi(k)$ invertible residue classes modulo $k$ is done in $O\left(\varphi(k) x^{2 / 3} / \ln ^{2} x\right)$ time, and therefore the computation time of the two values $\pi(x, 4,1)$ and $\pi(x, 4,3)$ is twice that of $\pi(x)$.

\section{Proof of TheOrem 1}

We now explain the method we used to compute $\pi(x, k, l)$ for large values of $x$. It is the natural adaptation of the method used in DR96; in particular the total time complexity is the same (for a fixed $k$ and $l$ ). From now on, we assume that $k$ is fixed and we write $\pi(x, l)$ instead of $\pi(x, k, l)$.

Let $y$ be a real positive number and let $T(x, y, l)$ be the set of positive integers $n$ such that

$$
\left\{\begin{array}{l}
n \leq x, \\
n \equiv l \quad(\bmod k), \\
p \mid n \Rightarrow p>y .
\end{array}\right.
$$

Assume that $y$ is such that $x^{1 / 3} \leq y \leq x^{1 / 2}$. Then each element $n$ of $T(x, y, l)$ has at most two (not necessarily distinct) prime factors. Thus we can split this set into three disjoint subsets $T_{0}(x, y, l), T_{1}(x, y, l)$, and $T_{2}(x, y, l)$, according to the number of (not necessarily distinct) prime factors. 
Let $F(x, y, l)$ be the cardinality of $T(x, y, l)$. The set $T_{0}(x, y, l)$ contains only 1 (resp. is empty) if $l=1$ (resp. $l \neq 1$ ). Its cardinality is thus $\delta_{l, 1}$. The set $T_{1}(x, y, l)$ contains all the prime numbers $p$ with $y<p \leq x$ and $p \equiv l(\bmod k)$. Therefore, its cardinality is $\pi(x, l)-\pi(y, l)$. Finally, let $P_{2}(x, y, l)$ denote the cardinality of $T_{2}(x, y, l)$. Putting everything together and rearranging terms, we get

$$
\pi(x, l)=F(x, y, l)-\delta_{l, 1}+\pi(y, l)-P_{2}(x, y, l) .
$$

2.1. Computation of $P_{2}(x, y, l)$. We have

$$
\begin{aligned}
P_{2}(x, y, l) & =\sum_{y<p \leq x^{1 / 2}} \sum_{\substack{p \leq q \leq x / p \\
p q \equiv l(\bmod k)}} 1 \\
& =\sum_{y<p \leq x^{1 / 2}}\left[\pi\left(x / p, l p^{-1}\right)-\pi\left(p-1, l p^{-1}\right)\right] \\
& =\sum_{y<p \leq x^{1 / 2}} \pi\left(x / p, l p^{-1}\right)-\sum_{y<p \leq x^{1 / 2}} \pi\left(p-1, l p^{-1}\right)
\end{aligned}
$$

with the implicit convention that $\pi\left(a, l p^{-1}\right)=\pi(a, n)$ with $n \equiv l p^{-1}(\bmod k)$.

We use an auxiliary sieve to obtain all primes up to $x^{1 / 2}$ and a parallel sieve of all invertible classes modulo $k$ up to $x / y$ to get the value of $\pi(x / p, n)$. We thus compute the first sum of equation (2.2) in time $O((x / y) \log \log x)$.

The second sum in (2.2) is computed directly using the primes $p$ coming from the auxiliary sieve. The computation time is $O\left(x^{\frac{1}{2}+\epsilon}\right)$, which is negligible compared to $O\left(x^{\frac{2}{3}} / \ln ^{2} x\right)$.

2.2. Computation of $\pi(y, l)$. We compute a table of all the prime numbers up to $y$ partitioned according to their class modulo $k$ using a sieve. The value of $\pi(y, n)$ for all classes $n$ invertible modulo $k$ is deduced directly from this table. This table and the values $\pi(x, n)$ will prove useful later. This can be done in $O(y \ln y)$ time, which is again negligible compared to $O\left(x^{\frac{2}{3}} / \ln ^{2} x\right)$.

2.3. Computation of $F(x, y, l)$. Recall that $F(x, y, l)$ counts the number of elements in $T(x, y, l)$. Let us number the prime numbers $p_{1}=2, p_{2}=3, \ldots$ For a positive integer $a$, let $\tilde{T}(x, a, l)=T\left(x, p_{a}, l\right)$ and $\tilde{F}(x, a, l)=F\left(x, p_{a}, l\right)$. Thus, $F(x, y, l)=\tilde{F}(x, a, l)$ where $a$ is the largest index such that $p_{a} \leq y$. We also set $\tilde{T}(x, 0, l)=T(x, 0, l)$ and $\tilde{F}(x, 0, l)=F(x, 0, l)$.

Now, we split the elements of $\tilde{T}(x, a, l)$ into two subsets: the first one containing those which are divisible by $p_{a+1}$ and the second containing those which are not. Clearly, the cardinality of the first set is $\tilde{F}\left(x / p_{a+1}, a, l p_{a+1}^{-1}\right)$ and the cardinality of the second is $\tilde{F}(x, a+1, l)$. We have proved the induction formula

$$
\tilde{F}(x, a+1, l)=\tilde{F}(x, a, l)-\tilde{F}\left(x / p_{a+1}, a, l p_{a+1}^{-1}\right) .
$$

Together with the initial conditions

$$
\tilde{F}(x, 0, l)=\left\lceil\frac{x+1-l}{k}\right\rceil \text { and } \quad \tilde{F}(x, a, l)=0 \text { whenever } x<1,
$$

we could use equation (2.3) to compute $F(x, y, l)$. However, such a method would require more than $x^{1-\varepsilon}$ time. 
Another extreme method would be to sieve all the positive integers congruent to $l$ modulo $k$ up to $x$ by all the prime numbers up to $y$ and count what is left. But, this is even worse since that would take more than $x \log \log x$ time.

In fact, the best way to compute $F(x, y, l)$ is to use a mix between these two methods as was already done in [LMO85 p. 542]. Let $z \geq y$ be a real number. Using the induction formula (2.3) to unfold the terms $F(x / m, p, n)$ while $m \leq z$ and $p \geq 2$, we get an expression with terms of the form $F(u, 0, n)$ which are easily computed and terms of the form $F(u, p, n)$ with $u<x / z$ which can be computed using a sieve up to $x / z$ (instead of $x$ in a "sieve only" method). More precisely, we get the formula

$$
F(x, y, l)=S_{0}+S
$$

with

$$
\begin{aligned}
S_{0}=\sum_{\substack{m \leq z \\
\gamma(m) \leq y}} \mu(m) \tilde{F}\left(\frac{x}{m}, 0, l m^{-1}\right), \\
S=-\sum_{b<a} \sum_{\substack{m \leq z<m p_{b} \\
\delta(m)>p_{b} \\
\gamma(m) \leq y}} \mu(m) \tilde{F}\left(\frac{x}{m p_{b}}, b-1, l\left(m p_{b}\right)^{-1}\right),
\end{aligned}
$$

where $\delta(m)$ (resp. $\gamma(m)$ ) denotes the smallest (resp. largest) prime number dividing $m$ if $m>1$, and $\delta(1)=\gamma(1)=1$.

2.4. Computation of $S$. We split the sum (recall that $a$ is the largest integer such that $\left.p_{a} \leq y\right)$

$$
S=-\sum_{p_{b}<y} \sum_{\substack{m \leq z<m p_{b} \\ \delta(m)>p_{b} \\ \gamma(m) \leq y}} \mu(m) F\left(\frac{x}{m p_{b}}, p_{b-1}, l\left(m p_{b}\right)^{-1}\right)=S_{1}+S_{2}+S_{3}
$$

into three parts according to the size of $p_{b}$ :

$$
\begin{aligned}
& S_{1}=-\sum_{x^{1 / 3}<p_{b}<y} \sum_{\substack{m \leq z<m p_{b} \\
\delta(m)>p_{b} \\
\gamma(m) \leq y}} \mu(m) F\left(\frac{x}{m p_{b}}, p_{b-1}, l\left(m p_{b}\right)^{-1}\right), \\
& S_{2}=-\sum_{x^{1 / 4}<p_{b} \leq x^{1 / 3}} \sum_{\substack{m \leq z<m p_{b} \\
\delta(m)>p_{b} \\
\gamma(m) \leq y}} \mu(m) F\left(\frac{x}{m p_{b}}, p_{b-1}, l\left(m p_{b}\right)^{-1}\right), \\
& S_{3}=-\sum_{p_{b} \leq x^{1 / 4}} \mu(m) F\left(\frac{x}{m p_{b}}, p_{b-1}, l\left(m p_{b}\right)^{-1}\right) . \\
& \substack{m \leq z<m p_{b} \\
\gamma(m)>p_{b} \\
\gamma(m) \leq y}
\end{aligned}
$$

The sum $S_{1}$ is easy to deal with. For each $p_{b}$ and each $m$, we have $m p_{b}>x^{2 / 3}$, SO

$$
\frac{x}{m p_{b}}<x^{1 / 3}<p_{b}
$$


and therefore

$$
F\left(\frac{x}{m p_{b}}, p_{b-1}, l\left(m p_{b}\right)^{-1}\right)= \begin{cases}1 & \text { if } l\left(m p_{b}\right)^{-1}=1 \\ 0 & \text { else }\end{cases}
$$

since $T\left(x /\left(m p_{b}\right), b-1, l(m p)^{-1}\right)$ is respectively $\{1\}$ or $\emptyset$.

Furthermore, note that $m$ is prime since all its prime factors are larger than $p_{b}>x^{1 / 3}$ and $m \leq z \leq x^{1 / 2}$. Thus, $\mu(m)$ is always equal to -1 and $S_{1}$ actually counts the primes congruent to $l p_{b}^{-1}$ modulo $k$ :

$$
S_{1}=\sum_{x^{1 / 3}<p_{b}<y} \sum_{\substack{p_{b}<q \leq y \\ q \equiv l p_{b}^{-1}(\bmod k)}} 1 .
$$

The sum $S_{1}$ is computed in negligible time $O(y)$.

Consider the sum $S_{2}$. Reasoning as above, it is clear that $m$ is a prime number. Therefore, we will write $q$ instead of $m$ to emphasize this fact. We get

$$
S_{2}=\sum_{x^{1 / 4}<p_{b} \leq x^{1 / 3}} \sum_{p_{b}<q \leq y} F\left(\frac{x}{q p_{b}}, p_{b-1}, l\left(q p_{b}\right)^{-1}\right) .
$$

Let $u$ be an element of $T\left(x /\left(q p_{b}\right), p_{b-1}, l\left(q p_{b}\right)^{-1}\right)$. Then $u$ has at most one prime factor since all its prime factors must be larger than or equal to $p_{b}>x^{1 / 4}$, and, on the other hand, $u$ must be smaller than $x /\left(q p_{b}\right) \leq x^{1 / 2}$. Thus, $u$ must be a prime unless $l \equiv q p_{b}(\bmod k)$ in which case $u=1$ is also valid. So, we get the formula (writing simply $p$ instead of $p_{b}$ ):

$$
S_{2}=\sum_{x^{1 / 4}<p \leq x^{1 / 3}} \sum_{p<q \leq y}\left[\max \left\{\pi\left(\frac{x}{q p}, l(q p)^{-1}\right)-\pi\left(p-1, l(q p)^{-1}\right), 0\right\}+\delta_{q p, l}\right]
$$

where $\delta_{q p, l}$ equals 1 if $q p \equiv l(\bmod k)$ and 0 otherwise. The $\max$ in the sum is due to the fact that, whenever $\pi\left(x /(q p), l(q p)^{-1}\right)-\pi\left(p-1, l(q p)^{-1}\right)<0$, the corresponding set $T\left(x /(q p), p-1, l(q p)^{-1}\right)$ contains only 1 if $q p \equiv l(\bmod k)$ and is empty otherwise.

We split this sum again:

$$
S_{2}=U_{1}+U_{2}+U_{3}
$$

with (note that the max condition translates to the fact that $q<x / p^{2}$ )

$$
\begin{aligned}
U_{1} & =\sum_{x^{1 / 4}<p \leq x^{1 / 3}} \sum_{p<q \leq \min \left\{y, x / p^{2}\right\}} \pi\left(\frac{x}{q p}, l(q p)^{-1}\right), \\
U_{2} & =\sum_{x^{1 / 4}<p \leq x^{1 / 3}} \sum_{p<q \leq y} \delta_{q p, l}, \\
U_{3} & =-\sum_{x^{1 / 4}<p \leq x^{1 / 3}} \sum_{p<q \leq \min \left\{y, x / p^{2}\right\}} \pi\left(p-1, l(q p)^{-1}\right) .
\end{aligned}
$$


We rewrite the sums $U_{2}$ and $U_{3}$ in the following way:

$$
\begin{aligned}
U_{2}= & \sum_{\substack{1 \leq m<k \\
(m, k)=1}} \sum_{\substack{x^{1 / 4}<p \leq x^{1 / 3} \\
p \equiv m(\bmod k)}} \sum_{\substack{p<q \leq y \\
q \equiv l m^{-1}(\bmod k)}} 1 \\
= & \sum_{\substack{1 \leq m<k \\
(m, k)=1}} \sum_{\substack{x^{1 / 4}<p \leq x^{1 / 3} \\
p \equiv m(\bmod k)}}\left[\pi\left(y, l m^{-1}\right)-\pi\left(p, l m^{-1}\right)\right] \\
= & \sum_{\substack{1 \leq m<k \\
(m, k)=1}} \pi\left(y, l m^{-1}\right)\left[\pi\left(x^{1 / 3}, m\right)-\pi\left(x^{1 / 4}, m\right)\right] \\
& -\sum_{x^{1 / 4}<p \leq x^{1 / 3}} \pi\left(p, l p^{-1}\right)
\end{aligned}
$$

and, letting $y(p)$ denote the minimum between $y$ and $x / p^{2}$ :

$$
\begin{aligned}
U_{3}=- & \sum_{\substack{1 \leq m<k \\
(m, k)=1}} \sum_{x^{1 / 4}<p \leq x^{1 / 3}} \sum_{\substack{p<q \leq y(p) \\
q \equiv l m^{-1}(\bmod k)}} \pi\left(p-1, m p^{-1}\right) \\
=- & \sum_{\substack{1 \leq m<k \\
(m, k)=1}} \sum_{x^{1 / 4}<p \leq x^{1 / 3}} \\
& \times \pi\left(p-1, m p^{-1}\right)\left[\pi\left(y(p), l m^{-1}\right)-\pi\left(p, l m^{-1}\right)\right] .
\end{aligned}
$$

Each sum is computed in a negligible time $O\left(x^{1 / 3}\right)$ using the precomputed table of prime numbers sorted by congruences classes mentioned above.

The hard part of the computation of $F(x, y, l)$ is the computation of the sum $U_{1}$. We write

$$
\begin{aligned}
U_{1}= & \sum_{x^{1 / 4}<p \leq x^{1 / 3}} \sum_{p<q \leq \min \left\{y, x / p^{2}\right\}} \pi\left(\frac{x}{q p}, l(q p)^{-1}\right) \\
= & \sum_{x^{1 / 4}<p \leq(x / y)^{1 / 2}} \sum_{p<q \leq y} \pi\left(\frac{x}{q p}, l(q p)^{-1}\right) \\
& +\sum_{(x / y)^{1 / 2}<p \leq x^{1 / 3}} \sum_{p<q \leq x / p^{2}} \pi\left(\frac{x}{q p}, l(q p)^{-1}\right) \\
= & \sum_{x^{1 / 4}<p \leq x / y^{2}} \sum_{p<q \leq y} \pi\left(\frac{x}{q p}, l(q p)^{-1}\right) \\
& +\sum_{x / y^{2}<p \leq(x / y)^{1 / 2}} \sum_{p<q \leq y} \pi\left(\frac{x}{q p}, l(q p)^{-1}\right) \\
& +\sum_{(x / y)^{1 / 2}<p \leq x^{1 / 3}} \pi\left(\frac{x}{q p}, l(q p)^{-1}\right) \\
= & \sum_{1}+\left(W_{2}+W_{3}\right)+\left(W_{4}+W_{5}\right)
\end{aligned}
$$


with

$$
\begin{aligned}
& W_{1}=\sum_{x^{1 / 4}<p \leq x / y^{2}} \sum_{p<q \leq y} \pi\left(\frac{x}{q p}, l(q p)^{-1}\right), \\
& W_{2}=\sum_{x / y^{2}<p \leq(x / y)^{1 / 2}} \sum_{p<q \leq(x / p)^{1 / 2}} \pi\left(\frac{x}{q p}, l(q p)^{-1}\right), \\
& W_{3}=\sum_{x / y^{2}<p \leq(x / y)^{1 / 2}} \sum_{(x / p)^{1 / 2}<q \leq y} \pi\left(\frac{x}{q p}, l(q p)^{-1}\right), \\
& W_{4}=\sum_{(x / y)^{1 / 2}<p \leq x^{1 / 3}} \sum_{p<q \leq(x / p)^{1 / 2}} \pi\left(\frac{x}{q p}, l(q p)^{-1}\right), \\
& W_{5}=\sum_{(x / y)^{1 / 2}<p \leq x^{1 / 3}} \sum_{\sqrt{x / p}<q \leq x / p^{2}} \pi\left(\frac{x}{q p}, l(q p)^{-1}\right) .
\end{aligned}
$$

The sums $W_{1}$ and $W_{2}$ are computed directly. Since $x / q p$ can be as large as $x^{1 / 2}$, we use a parallel sieve of all invertible classes modulo $k$ up to $x^{1 / 2}$ to get the values of $\pi\left(x /(q p), l(q p)^{-1}\right)$.

For $W_{3}$, since $q$ is larger than $(x / p)^{1 / 2}$, a large number of consecutive values of $q$ gives the same value of $\pi\left(x /(q p), l(q p)^{-1}\right)$; henceforth this sum can be evaluated more efficiently by grouping these consecutive values of $q$. The same technique applies to $W_{5}$.

Finally, the sum $W_{4}$ is computed using, once again, the precomputed table.

The exact time complexity of the computation of these sums is given in [DR96; in any case they are $O\left(x^{2 / 3} / \log ^{2} x\right)$.

\section{NUMERICAL RESULtS}

We have implemented the method described above in $\mathrm{C}++$ on a DEC Alpha EV6 $500 \mathrm{MHz}$ and a Pentium III $1 \mathrm{GHz}$. We have computed the values of $\pi(x, 4,1)$ and $\pi(x, 4,3)$ for $x=d \cdot 10^{j}$ with $1 \leq d \leq 9$ and $10 \leq j \leq 19$ and also for $x=10^{20}$. These values are given in Table 2

We have also made a thorough search of regions where $\delta(x)$ is negative for most of the values of $x$. Indeed, equation (1.1) shows that a search with a step of 0.004 on a logarithmic scale for a large interval of values of $x$ would hit values $x$ for which $\delta(x)<0$ with a good chance. We performed a computation of the values of $\delta\left(x_{0} \times r^{n}\right)$ for $x_{0}=1,000$ and $r=1.004$, up to $x=1,088,537,721,123,564,252$ (as far as today). When the value of $\delta(x)$ obtained was positive but relatively small, we computed several values of $\delta$ near $x$ to see whether or not there was a region in the area. This method led to the rediscovery of all the previous regions already known (see Table 1) and also to the discovery of a new region around $x=10^{18}$. Note that 


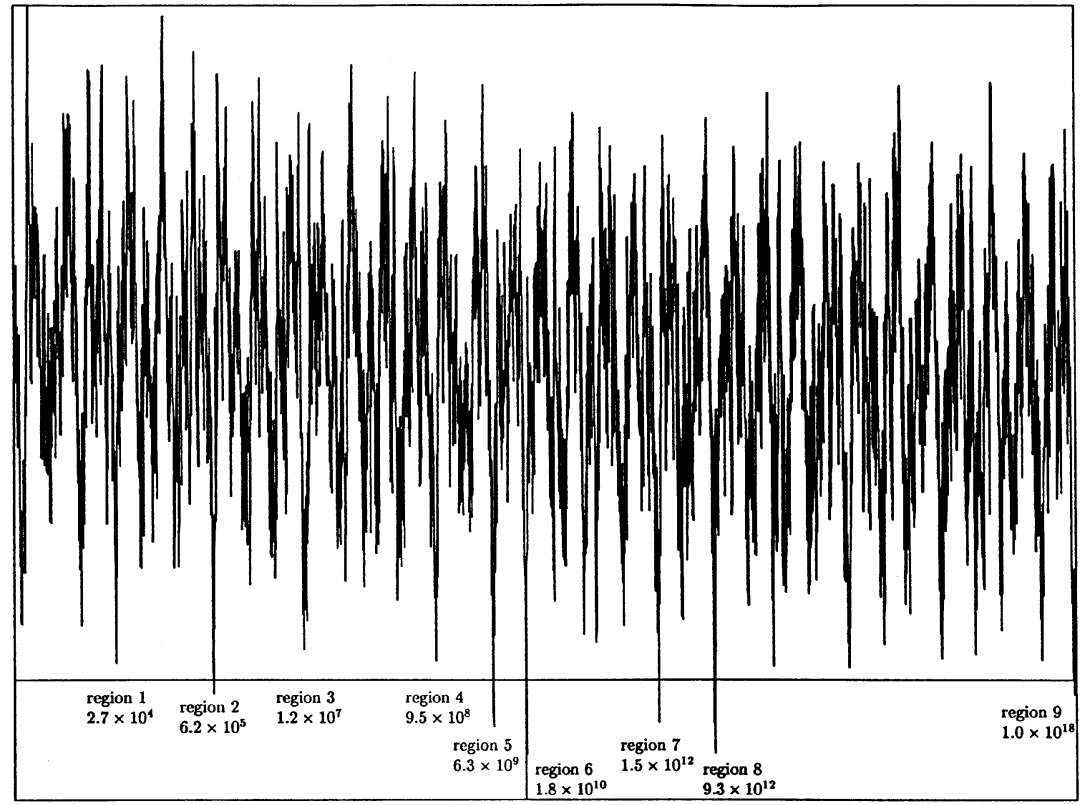

FiguRE 1. $(\log (x), \delta(x) \log (x) / \sqrt{(x)})$

we do not claim this search to be exhaustive since the method may miss narrow regions. Figure 1 gives a graph of these computations. On the horizontal axis are the values of $x$ on a logarithmic scale, and on the vertical axis are the values of $\delta(x) \log (x) / \sqrt{x}$.

The new region extends as far as $1.005 \times 10^{18}$, so it surrounds $10^{18}$. It should be noted that $10^{18}$ does not belong to $\Delta^{-}$, but still the value of $\delta\left(10^{18}\right)$ is relatively small.

TABLE 1. Known regions where $\delta(x)<0$

\begin{tabular}{cll} 
Region & Starts at & \\
\hline 1 & 26,861 & Leech [Lee57], 1957 \\
2 & $6.16 \times 10^{5}$ & Leech [Lee57], 1957 \\
3 & $1.23 \times 10^{7}$ & Lehmer, 1969 \\
4 & $9.51 \times 10^{8}$ & Lehmer, 1969 \\
5 & $6.31 \times 10^{9}$ & Bays and Hudson [BH78], 1979 \\
6 & $1.85 \times 10^{10}$ & Bays and Hudson [BH78], 1979 \\
7 & $1.49 \times 10^{12}$ & Bays and Hudson, 1996 \\
8 & $9.32 \times 10^{12}$ & Bays et al. [BFHR01], 2001 \\
9 & $9.97 \times 10^{17}$ & \\
\hline
\end{tabular}


TABle 2.

\begin{tabular}{l|rrr}
$x$ & $\pi(x, 4,1)$ & $\pi(x, 4,3)$ & $\delta(x)$ \\
\hline $1 \times 10^{10}$ & 227523275 & 227529235 & 5960 \\
$2 \times 10^{10}$ & 441101890 & 441104825 & 2935 \\
$3 \times 10^{10}$ & 649997354 & 650008571 & 11217 \\
$4 \times 10^{10}$ & 855972440 & 855982992 & 10552 \\
$5 \times 10^{10}$ & 1059822165 & 1059832412 & 10247 \\
$6 \times 10^{10}$ & 1262014995 & 1262023159 & 8164 \\
$7 \times 10^{10}$ & 1462847357 & 1462852181 & 4824 \\
$8 \times 10^{10}$ & 1662521926 & 1662537319 & 15393 \\
$9 \times 10^{10}$ & 1861205914 & 1861223076 & 17162 \\
$1 \times 10^{11}$ & 2059020280 & 2059034532 & 14252 \\
$2 \times 10^{11}$ & 4003548492 & 4003556566 & 8074 \\
$3 \times 10^{11}$ & 5909207980 & 5909231154 & 23174 \\
$4 \times 10^{11}$ & 7790493403 & 7790512253 & 18850 \\
$5 \times 10^{11}$ & 9654058131 & 9654078010 & 19879 \\
$6 \times 10^{11}$ & 11503736012 & 11503765773 & 29761 \\
$7 \times 10^{11}$ & 13342013346 & 13342060963 & 47617 \\
$8 \times 10^{11}$ & 15170671955 & 15170711571 & 39616 \\
$9 \times 10^{11}$ & 16990975120 & 16991012465 & 37345 \\
$1 \times 10^{12}$ & 18803924340 & 18803987677 & 63337 \\
$2 \times 10^{12}$ & 36650920051 & 36650976087 & 56036 \\
$3 \times 10^{12}$ & 54170123581 & 54170175121 & 51540 \\
$4 \times 10^{12}$ & 71483076254 & 71483131871 & 55617 \\
$5 \times 10^{12}$ & 88645790439 & 88645871209 & 80770 \\
$6 \times 10^{12}$ & 105690668569 & 105690758469 & 89900 \\
$7 \times 10^{12}$ & 122638762289 & 122638926514 & 164225 \\
$8 \times 10^{12}$ & 139504962196 & 139505108614 & 146418 \\
$9 \times 10^{12}$ & 156300160163 & 156300193944 & 33781 \\
$1 \times 10^{13}$ & 173032709183 & 173032827655 & 118472 \\
$2 \times 10^{13}$ & 337947869842 & 337948039428 & 169586 \\
$3 \times 10^{13}$ & 500060778623 & 500060890229 & 111606 \\
$4 \times 10^{13}$ & 660405866854 & 660406104847 & 237993 \\
$5 \times 10^{13}$ & 819461739349 & 819462025217 & 285868 \\
$6 \times 10^{13}$ & 977505071501 & 977505356756 & 285255 \\
$7 \times 10^{13}$ & 1134716310961 & 1134716560342 & 249381 \\
$8 \times 10^{13}$ & 1291221836521 & 1291222276965 & 440444 \\
$9 \times 10^{13}$ & 1447116002078 & 1447116248704 & 246626 \\
$1 \times 10^{14}$ & 1602470783672 & 1602470967129 & 183457 \\
$2 \times 10^{14}$ & 3135212239502 & 3135212411812 & 172310 \\
$3 \times 10^{14}$ & 4643720595358 & 4643721004921 & 409563 \\
$4 \times 10^{14}$ & 6136911872530 & 6136912282960 & 410430 \\
$5 \times 10^{14}$ & 7618916303080 & 7618917351539 & 1048459 \\
$6 \times 10^{14}$ & 9092127220696 & 9092128070873 & 850177 \\
$7 \times 10^{14}$ & 10558104318534 & 10558104592488 & 273954 \\
$8 \times 10^{14}$ & 12017944798977 & 12017945569183 & 770206 \\
$9 \times 10^{14}$ & 13472462653549 & 13472463812671 & 1159122
\end{tabular}


Table 2 (continued)

\begin{tabular}{|c|c|c|c|}
\hline$x$ & $\pi(x, 4,1)$ & $\pi(x, 4,3)$ & $\delta(x)$ \\
\hline $1 \times 10^{15}$ & 14922284735484 & 14922285687184 & 951700 \\
\hline $2 \times 10^{15}$ & 29239107639569 & 29239108042321 & 402752 \\
\hline $3 \times 10^{15}$ & 43344300693083 & 43344302117035 & 1423952 \\
\hline $4 \times 10^{15}$ & 57315493601108 & 57315495302891 & 1701783 \\
\hline $5 \times 10$ & 71188707903700 & 71188709292663 & 1388963 \\
\hline $6 \times 10$ & 84984830526287 & 84984832028263 & 1501976 \\
\hline $7 \times 10$ & 98717495795309 & 98717498283021 & 2487712 \\
\hline $8 \times 10$ & 112396302108982 & 112396304209617 & 2100635 \\
\hline $9 \times 1$ & 8365161887 & 8368292040 & 3130153 \\
\hline $1 \times 1$ & 16878 & 9172246129 & 3458334 \\
\hline $2 \times 1$ & 7128 & 17190 & 6210367 \\
\hline $3 \times$ & 359 & 0140 & 4918380 \\
\hline $4 \times$ & 72 & 377 & 7149605 \\
\hline $5 \times$ & 98 & 272 & 4782574 \\
\hline $6 \times$ & 85 & 7053 & 7983503 \\
\hline $7 \times 1$ & 11 & 08 & 10712397 \\
\hline $8 \times 1$ & 23 & 1055 & 10518397 \\
\hline $9 \times 1$ & 38 & 661 & 7474773 \\
\hline $2 \times 1$ & & 702 & 0713497 \\
\hline $3 \times 1$ & & 339 & 3539876 \\
\hline $4 \times 1$ & & 478 & 5987635 \\
\hline $5 \times 1$ & 33 & 6292 & 21159153 \\
\hline $6 \times 1$ & 33 & 786 & 5651753 \\
\hline $7 \times 1$ & 90 & 65 & 3605875 \\
\hline $8 \times 10$ & 12670 & 6809 & 3786554 \\
\hline $9 \times 10$ & 46009 & 1162094630 & 29535678 \\
\hline $1 \times 10$ & 584 & 2369977145 & 2581691 \\
\hline $2 \times 1$ & 06238 & 24322580657 & 33978354 \\
\hline $3 \times 10$ & 12735239102 & 36127352406660798 & 15634514 \\
\hline $4 \times 10$ & 3041673 & 478381304871 & 70415398 \\
\hline $5 \times 1$ & 947986 & 59479994889656049 & 91038627 \\
\hline $6 \times 10$ & 06752467849 & 71067524734130848 & 55639553 \\
\hline $7 \times 10$ & 69794 & 826102570015 & 22133695 \\
\hline $8 \times 1$ & 114914605549098 & 4114914641880405 & 36331307 \\
\hline $9 \times 1$ & 5586489592518919 & 5586489650739358 & 58220439 \\
\hline $1 \times 1$ & 7028833597800689 & 17028833678543917 & 80743228 \\
\hline $2 \times 1$ & 27545992966 & 230318827580012523 & 34019557 \\
\hline $3 \times 1$ & 2279960248880580 & 342279960334204109 & 5323529 \\
\hline $4 \times 10$ & 33052574434 & 4533052576621 & 18728354 \\
\hline $5 \times 1$ & 581 & 563889961936366961 & 82549380 \\
\hline & & & \\
\hline & & 782406420248 & 171614523 \\
\hline & & & \\
\hline & & & \\
\hline $1 \times 10^{20}$ & 1110409801150582707 & 1110409801410336132 & \\
\hline
\end{tabular}




\section{REFERENCES}

[BH78] C. Bays and R. H. Hudson, On the fluctuations of Littlewood for primes of the form $4 n \neq 1$, Math. Comp. 32 (1978), 281-286. MR 57:16174

[BFHR01] C. Bays, K. Ford, R. H. Hudson and M. Rubinstein, Zeros of Dirichlet L-functions near the real axis and Chebyshev's bias, J. Number Theory 87 (2001), 54-76. MR 2001m:11148

[DR96] M. Deléglise and J Rivat, Computing $\pi(x)$ : the Meissel, Lehmer, Lagarias, Miller, Odlyzko method, Math. Comp. 65 (1996), 235-245. MR 96d:11139

[FM00] A. Feuerverger and G. Martin, Biases in the Shanks-Rényi prime number race, Experiment. Math. 9 (2000), 535-570. MR 2002d:11111

[Gou01] X. Gourdon, http://numbers.computation.free.fr/Constants/Primes/Pix/

[Ing90] A. E. Ingham, The distribution of prime numbers, Cambridge University Press, 1990. MR 91f: 11064

[LMO85] J. C. Lagarias, V. S. Miller and A. M. Odlyzko, Computing $\pi(x)$ : the Meissel-Lehmer method, Math. Comp. 44 (1985), 537-560. MR 86h:11111

[Lee57] J. Leech, Note on the distribution of prime numbers, J. London Math. Soc. 32 (1957), 56-58. MR 18:642d

[Leh59] D. H. Lehmer, On the exact number of primes less than a given limit, Illinois J. Math. 3 (1959), 381-388. MR 21:5613

[Lit14] J. E. Littlewood, Sur la distribution des nombres premiers, C. R. Acad. Sci. Paris 158 (1914), 358-372.

[RS94] M. Rubinstein and P. Sarnak, Chebyshev's Bias, Experiment. Math. 3 (1994), 173-197. MR 96d:11099

Institut Girard Desargues, Université Lyon I, 21, avenue Claude Bernard, 69622 Villeurbanne Cedex, France

E-mail address: Marc.Deleglise@igd.univ-lyon1.fr

LaCo, Département de Mathématiques, avenue Albert Thomas, 87060 Limoges Cedex, FRANCE

E-mail address: dusart@unilim.fr

Institut Girard Desargues, Université Lyon I, 21, avenue Claude Bernard, 69622 Villeurbanne Cedex, France

E-mail address: Xavier.Roblot@igd.univ-lyon1.fr 